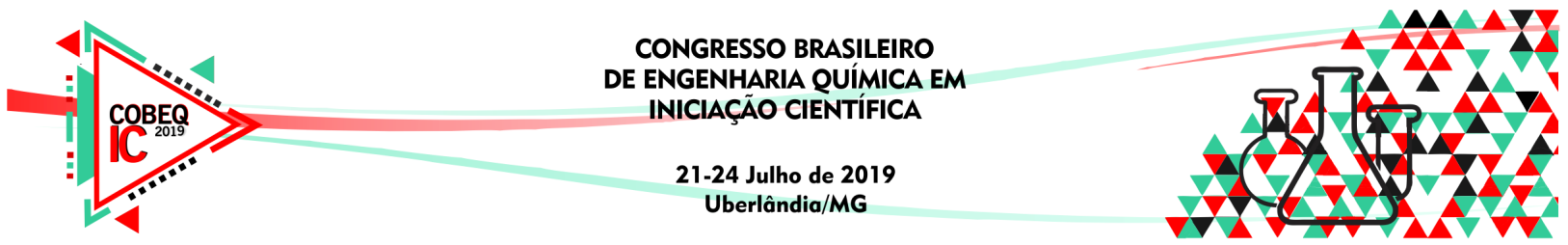

\title{
ESTUDO VIA CFD DA SEGREGAÇÃO DE PARTÍCULAS DE DIFERENTES DENSIDADES EM UM LEITO DE JORRO
}

\author{
G. H. S. PAIVA ${ }^{1}$, M. B. F. SILVA ${ }^{1}$ e I. C. BICALHO ${ }^{1}$ \\ ${ }^{1}$ Universidade Federal de Lavras, Engenharia Química \\ E-mail para contato: marcusbfs@gmail.com
}

\begin{abstract}
RESUMO - O leito de jorro tem sido estudado em diversas aplicações, tais como secagem de materiais, granulação e recobrimento de partículas, pirólise, dentre outros. Isto se deve ao fato do equipamento fornecer altas taxas de circulação de partículas juntamente com um excelente contato fluido-partícula, o que favorece a obtenção de elevados coeficientes de transferência calor e massa. $\mathrm{O}$ conhecimento da fluidodinâmica do equipamento é de suma importância para melhorar o seu desempenho, adaptá-lo para novas aplicações, bem como entender suas limitações. As técnicas de Fluidodinâmica Computacional (CFD) consistem na solução numérica das equações de conservação (massa, momentum, energia) e permitem obter maiores detalhes da fluidodinâmica de diversos equipamentos. Este trabalho tem como objetivo utilizar técnicas de CFD para simular o comportamento fluidodinâmico (perfis de velocidade e porosidade) e estimar a queda de pressão em um leito de jorro operando com mistura de partículas. Paralelamente, foi avaliada a capacidade de predição da ferramenta CFD, através da comparação dos resultados simulados com dados experimentais reportados na literatura.
\end{abstract}

\section{INTRODUÇÃO}

O leito de jorro possui aplicação ligada a processos que requerem um contato fluido partícula eficiente, tais como processos de secagem (Brito et al., 2015; Delmiro et al., 2015), recobrimento de partículas (Almeida e Rocha, 2008; Westphalen et al., 2015) e pirólise (Covre et al., 2014; Ferreira et al., 2017). A dinâmica das partículas presentes no leito depende das propriedades do sistema fluido-partícula, das características do escoamento do fluido e também da geometria do leito, como o bocal de alimentação e a angulação da parte cônica.

As técnicas de CFD (Computational Fluid Dynamics) utilizam métodos numéricos com o auxílio computacional para obter a solução aproximada das equações de conservação de massa, energia e momento. O estudo da fluidodinâmica de um leito de jorro utilizando CFD permite descrever a distribuição de velocidade de partículas e do fluido, perfil de porosidade e queda de pressão do leito (Duarte et al., 2005; Santos, 2008; He et al., 1994). De modo geral, esses estudos mostram que a ferramenta cumpre a finalidade de prever os fenômenos reais que ocorrem no leito, podendo complementar os estudos feitos experimentalmente. Dito isto, objetivou-se neste estudo simular um leito de jorro operando com mistura de partículas, baseado em estudos experimentais realizados por Campos et al. (2011). 


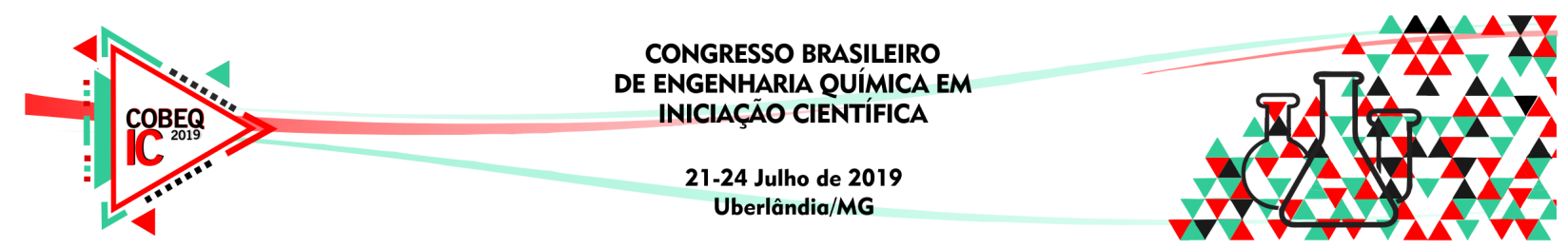

\section{MATERIAIS E MÉTODOS}

Para o estudo de caso de um leito de jorro operando com mistura de partículas, foi selecionado o trabalho de Campos et al. (2011). Neste caso, o leito usado era convencional cônico-cilíndrico (diâmetro da coluna de $0,21 \mathrm{~m}$; diâmetro da entrada de $0,035 \mathrm{~m}$; ângulo do cone $60^{\circ}$ e altura do leito de $0,85 \mathrm{~m}$ ) e a mistura multifásica foi composta por esferas de polietileno e de vidro.

As esferas de vidro possuíam diâmetro de $0,004 \mathrm{~m}$ e densidade de $2480 \mathrm{~kg} / \mathrm{m}^{3}$. As partículas de polietileno apresentavam diâmetro de $0,004 \mathrm{~m}$ e densidade $877,5 \mathrm{~kg} / \mathrm{m}^{3}$. Foram reproduzidas nas simulações a seguinte proporção de mistura, em frações mássicas: $25 \%$ de esferas de polietileno e $75 \%$ de esferas de vidro. As vazões de ar na entrada simuladas foram de 20 e $40 \mathrm{~m}^{3} / \mathrm{h}$.

Para realizar as simulações foi utilizado o software ANSYS Fluent ${ }^{\circledR}$ R19.0 Academic. As malhas utilizadas foram tridimensionais (3D) e hexaédricas. As simulações foram conduzidas em regime transiente utilizando o modelo euleriano multifásico. Foi utilizado o algoritmo Phase Coupled SIMPLE para o acoplamento pressão-velocidade, o modelo de Gidaspow para o arraste, Syamlal-Obrien para viscosidade granular e Lun-et-al para a pressão de sólidos. Como estratégia de discretização das componentes da equação de movimento optou-se pela escolha de esquemas de interpolação do tipo UPWIND de primeira ordem. Para os cálculos, o critério de convergência adotado para os resíduos foi de no máximo $1 \times 10^{-3}$ e o time step de $1 \times 10^{-4}$. Foram definidos 15000 time-steps o que correspondeu a um tempo de 15 segundos de simulação. As simulações foram feitas em um notebook DELL®, com um processador INTEL ${ }^{\circledR}$ Core $^{\mathrm{TM}}$ i5-2430M com 4 núcleos e 6 gigabytes de memória RAM.

Foi realizado um teste de independência de malha, com cinco diferentes densidades de malhas (162 mil, 216 mil, 243 mil, 302,4 e 348 mil células), para se averiguar a influência do número de células nos resultados simulados. Para este teste utilizou-se somente ar que era alimentado na entrada do leito no valor de $30 \mathrm{~m} / \mathrm{s}$.

\section{RESULTADOS E DISCUSSÕES}

Para o teste de independência de malha, foram observados os resultados de queda de pressão no leito, Figura 1. As quedas de pressão simuladas foram de $-184,83 \mathrm{~Pa},-141,16 \mathrm{~Pa}$, $-130,09 \mathrm{~Pa},-120,44 \mathrm{~Pa}$ e -115,02 Pa para as malhas de 162 mil, 216 mil, 243 mil, 302,4 e 348 mil células, respectivamente. Os resultados indicam uma tendência de estabilização da queda de pressão com o aumento do número de células, sendo que a diferença entre os valores fornecidos pelas malhas 4 e 5 situa-se abaixo de 5\%. Deste modo, a malha 4 (302,4 mil células) foi selecionada para realizar as simulações por requerer um menor esforço computacional.

Utilizando a malha de 302,4 mil células, foram feitas as simulações e obtidos os perfis de contorno de fração de sólidos num plano no interior do leito, e comparados com a reprodução do trabalho de Campos et al. (2011). Também foi feita a comparação dos 


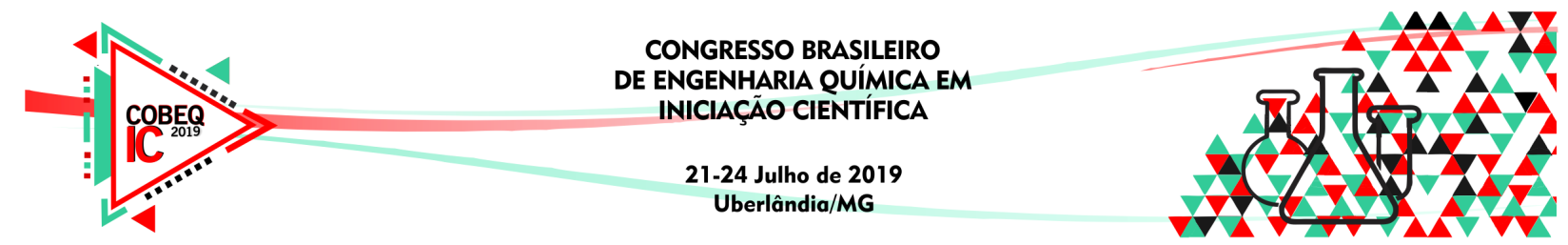

resultados experimentais de queda de pressão com base na entrada da alimentação, com os obtidos na simulação.

Na Figura 2 é representa a relação entre a curva característica do leito de jorro e os eventos visuais que ocorrem durante o jorro, obtido de Campos et al. (2011). As Figuras 3 e 4 mostram o perfil de fração de sólidos simulados para as esferas de polietileno e esferas de vidro obtidos para as vazões de ar na entrada de 20 e $40 \mathrm{~m}^{3} / \mathrm{h}$, respectivamente. Nestas figuras também é mostrado o perfil experimental obtido para permitir comparações.

Figura 1 - Teste de malha.

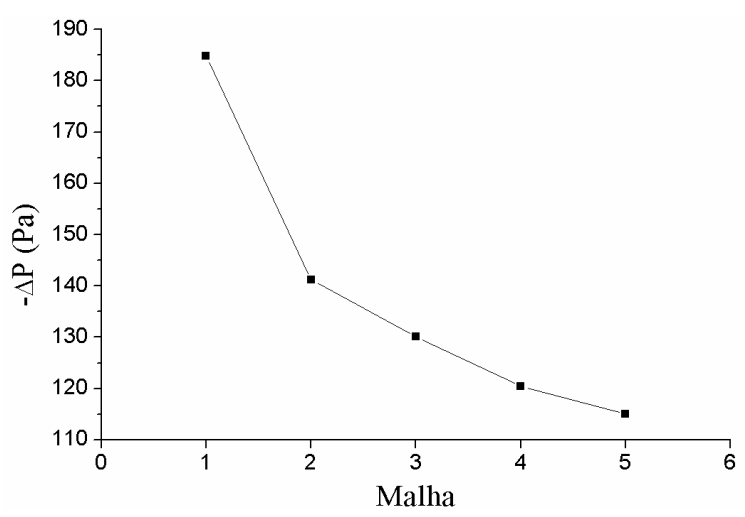

Figura 2 - Curva característica do leito de jorro e imagens dos perfis (Campos et al., 2011).

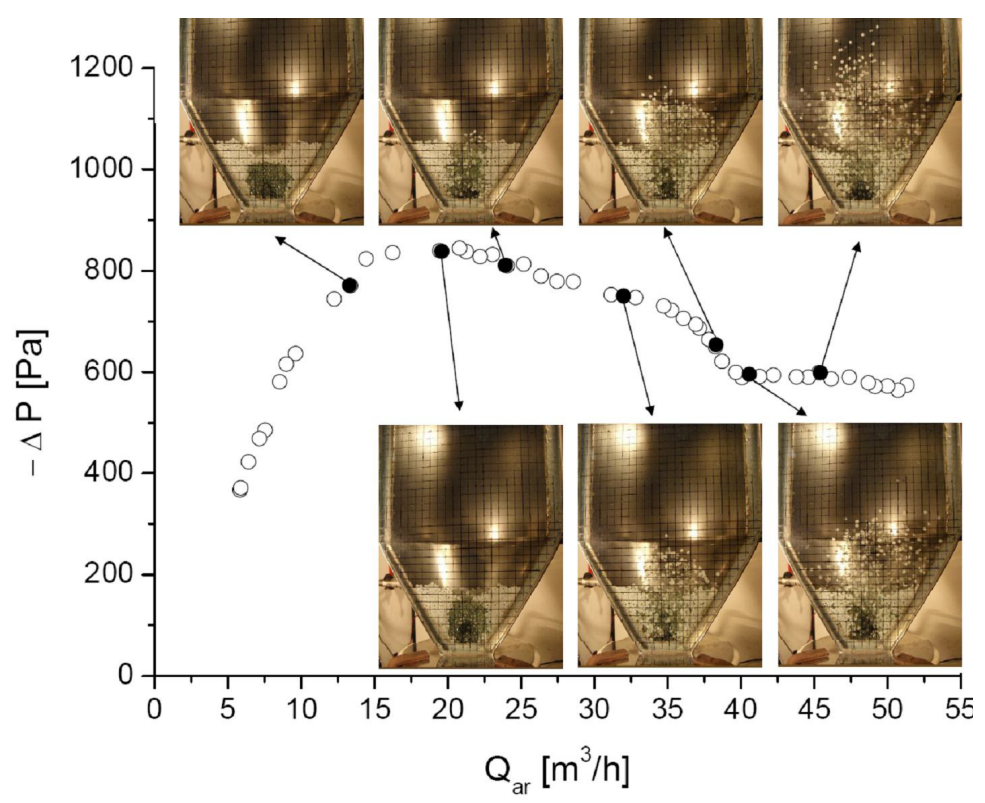

Pode-se afirmar que de maneira geral as simulações conseguiram reproduzir de forma razoável o comportamento experimental das partículas, como pode ser observado nos perfis de fração de sólidos mostrados nas Figuras 3 e 4 . Para as esferas de vidro e partículas de polietileno de mesmo tamanho a diferença na massa específica foi o fator que levou a uma segregação no leito, onde as partículas mais pesadas (esferas de vidro) situaram-se majoritariamente no fundo do leito. 


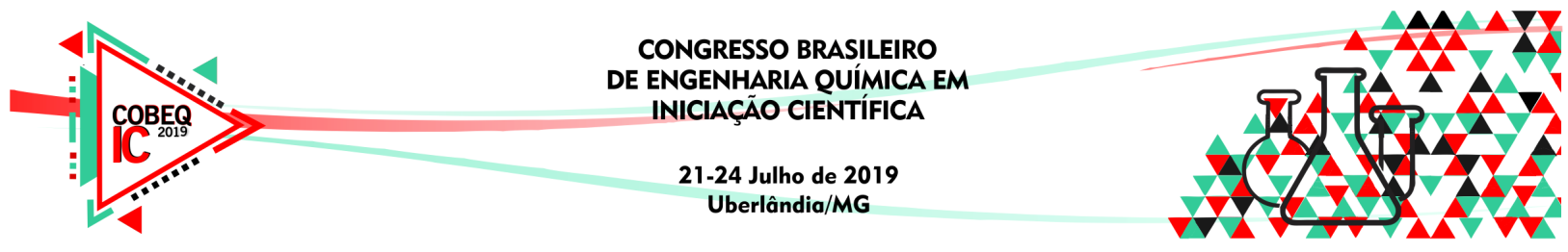

Figura 3 - Perfil de fração de sólidos simulados para a vazão de $20 \mathrm{~m} 3 / \mathrm{h}$.

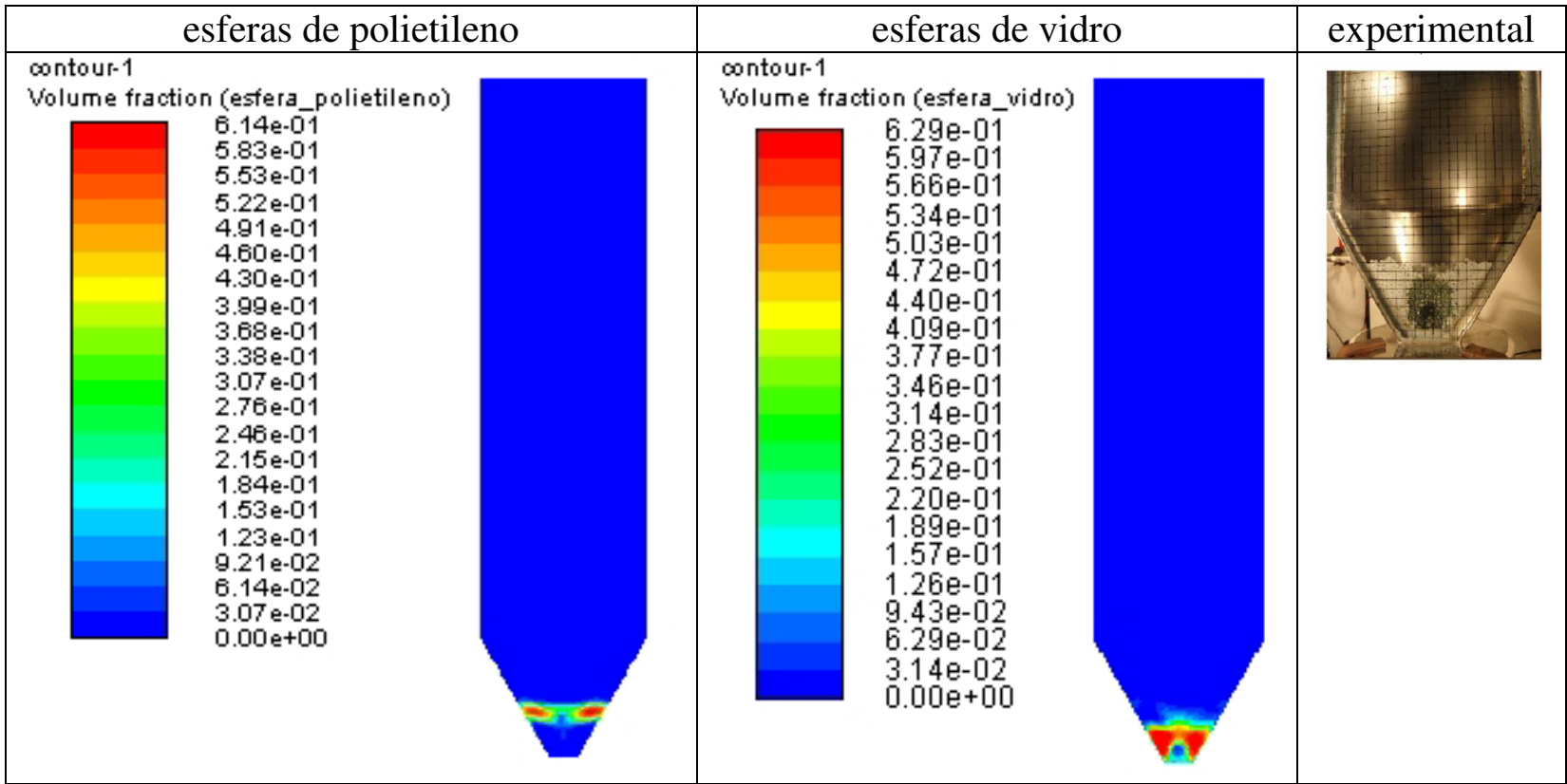

Figura 4 - Perfil de fração de sólidos simulados para a vazão de 40 m³/h.

\begin{tabular}{|c|c|c|}
\hline esferas de polietileno & esferas de vidro & experimental \\
\hline $\begin{array}{l}\text { Dontour-1 } \\
\text { Volume fraction (esfera_polietileno) } \\
\qquad \begin{array}{|c|c|c|}.00 \mathrm{e}-01 \\
5.70 \mathrm{e}-01 \\
5.40 \mathrm{e}-01 \\
5.10 \mathrm{e}-01 \\
4.80 \mathrm{e}-01 \\
4.50 \mathrm{e}-01 \\
4.20 \mathrm{e}-01 \\
3.90 \mathrm{e}-01 \\
3.60 \mathrm{e}-01 \\
3.30 \mathrm{e}-01 \\
3.00 \mathrm{e}-01 \\
2.70 \mathrm{e}-01 \\
2.40 \mathrm{e}-01 \\
2.10 \mathrm{e}-01 \\
1.80 \mathrm{e}-01 \\
1.50 \mathrm{e}-01 \\
1.20 \mathrm{e}-01 \\
9.00 \mathrm{e}-02 \\
6.00 \mathrm{e}-02 \\
3.00 \mathrm{e}-02 \\
0.00 \mathrm{e}+00\end{array}\end{array}$ & $\begin{array}{l}\text { contour-1 } \\
\text { Volume fraction (esfera_vidro) } \\
\begin{array}{|l}5.66 \mathrm{e}-01 \\
5.37 \mathrm{e}-01 \\
5.09 \mathrm{e}-01 \\
4.81 \mathrm{e}-01 \\
4.52 \mathrm{e}-01 \\
4.24 \mathrm{e}-01 \\
3.96 \mathrm{e}-01 \\
3.68 \mathrm{e}-01 \\
3.39 \mathrm{e}-01 \\
3.11 \mathrm{e}-01 \\
2.83 \mathrm{e}-01 \\
2.55 \mathrm{e}-01 \\
2.26 \mathrm{e}-01 \\
1.98 \mathrm{e}-01 \\
1.70 \mathrm{e}-01 \\
1.41 \mathrm{e}-01 \\
1.13 \mathrm{e}-01 \\
8.48 \mathrm{e}-02 \\
5.66 \mathrm{e}-02 \\
2.83 \mathrm{e}-02 \\
0.00 \mathrm{e}+00 \\
\end{array}\end{array}$ & $\frac{7}{(a+5)}$ \\
\hline
\end{tabular}

Na Tabela 1 podem ser observados os valores simulados de queda de pressão na base do leito e os valores experimentais obtidos por Campos et al. (2011). 


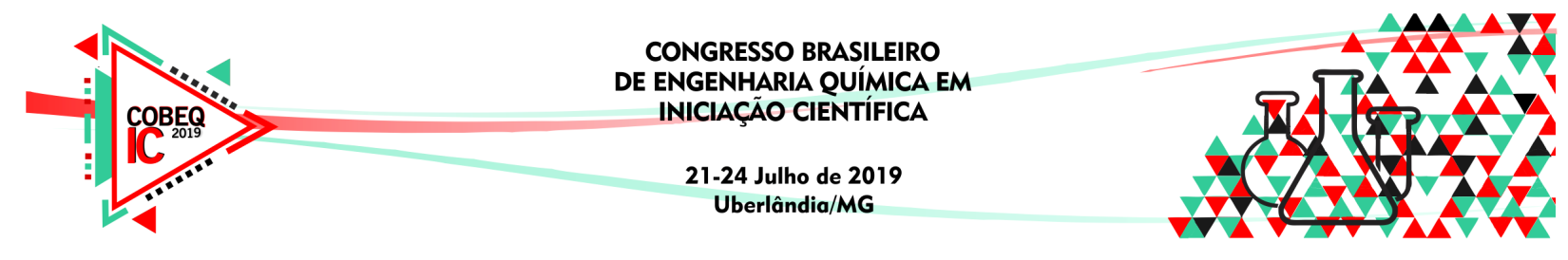

Tabela 1 - Queda de pressão simulada no leito e valor experimental.

\begin{tabular}{|c|c|c|c|}
\hline Vazão $\left(\mathrm{m}^{3} / \mathrm{h}\right)$ & $\begin{array}{c}\Delta P \text { simulada } \\
(\mathrm{Pa})\end{array}$ & $\begin{array}{c}\Delta P \text { experimental } \\
(\mathrm{Pa})\end{array}$ & Erro (\%) \\
\hline \hline 20 & 661,63 & 830 & 20,29 \\
\hline 40 & 528,77 & 600 & 11,87 \\
\hline
\end{tabular}

\section{CONCLUSÕES}

As simulações computacionais via CFD se mostraram capazes de representar a fluidodinâmica e distribuição dos sólidos no interior do leito, sendo possível a visualização do fenômeno de segregação de partículas de diferentes massas específicas. As quedas de pressão simuladas desviaram no máximo em torno de $20 \%$ do valor experimental, esses desvios são atribuídos a fatores como grau de refinamento da malha e critério de resíduos adotado.

\section{REFERÊNCIAS}

ALMEIDA, C. e ROCHA, S. C. S.. Eficiência do processo de recobrimento de sementes de brócolos recobertas com polímero em leito de jorro. Eng. Agríc. [online]. v. 28, n. 2, p. 305-314, 2008.

BRITO, R. C.; BATISTA, J. N. M.; PÁDUA, T.; FREIRE, J. T.; BÉTTEGA, R.. Estudo da secagem de sementes de sorgo em leito de jorro. Anais do Congresso Brasileiro de Engenharia Química - COBEQ 2016.

CAMPOS, A. V. P.; VIEIRA, B. C. N.; FRANCISQUETTI, M. C. C.; SANTANA, R.C.; BARROZO, M.A.S.. Fluidodinâmica de um leito de jorro operando com misturas de partículas de diferentes densidades. XVI Jornada em Engenharia Química, Universidade Federal de Uberlândia, 2011.

COVRE, V. R.; MELO, J. L. Z; BACELOS, M. S. Leito de jorro cônico composto de mistura de partículas de areia e compósito AL/PEBD. Blucher Chemical Engineering Proceedings, v. 1, n. 2, p. 5799-5806, 2015.

DELMIRO, T. M.; CARLOS, G. F.; PONTES JÚNIOR, S. M.. Secagem de misturas de açaí e banana verde em leito de jorro. Anais do Congresso Brasileiro de Engenharia Química - COBEQ 2016.

DUARTE, C. R.; MURATA, V. V.; BARROZO, M. A. S. A study of the fluid dynamics of the spouted bed using CFD. Brazilian Journal of Chemical Engineering, v. 22, n. 2, p. 263-270, 2005.

FERREIRA, C. G.; SANTOS, F. B.; BARCELOS, K. M.; RIBEIRO, D. C.; LIRA, T. S.. Estudo da fluidodinâmica de um leito de jorro aplicado a uma mistura de casca de coco e areia via análise experimental e simulação por CFD. Blucher Chemical Engineering Proceedings, v. 1, n. 4, p. 2446-8711, 2017. 


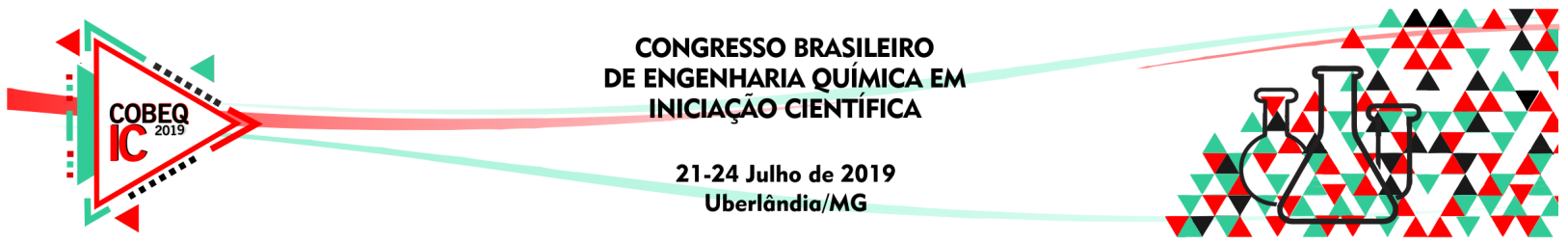

HE, Y. $\square$ L.; LIM, C. J.; GRACE, J. R.; ZHU, J. $\square$ X.; QZN, S. $\square$ Z.. Measurements of voidage profiles in spouted beds. The Canadian Journal of Chemical Engineering, v. 72, n. 2, p. 229-234, 1994.

SANTOS, K. G.. Estudo da fluidodinâmica do leito de jorro por CFD com malhas tridimensionais. Dissertação de mestrado. Universidade Federal de Uberlândia, 2008.

WESTPHALEN, G. F. DALLA NORA, F.; ZORZI, B.; ECHEVARRIA, E.; da COSTA, T. P.; ROSA, G. S.. Estudo da influência das condições operacionais na eficiência do recobrimento de ureia em leito de jorro. Blucher Chemical Engineering Proceedings, v. 1, n. 3, p. 2117-2122, 2015. 